\title{
Performance Analysis of Unslotted Fiber-Optic Code-Division Multiple-Access (CDMA) Packet Networks
}

\author{
Cherng-Shung Hsu, Member, IEEE, and Victor O. K. Li, Fellow, IEEE
}

\begin{abstract}
This paper examines code-division multiple-access (CDMA) techniques used in unslotted fiber-optic packet networks. Since the inherent properties and signal processing of the conventional communication channels are different from those of the fiber-optic channels, new code sequences must be constructed for fiber-optic applications. In unslotted systems, the exact solution is very difficult to obtain. Therefore, two approximation methods are presented to analyze the performance of such systems. Simulation is performed to verify the accuracy of the results.
\end{abstract}

\section{INTRODUCTION}

$\mathbf{M}$ OST of the present research on CDMA networks has been restricted to slotted systems. It is easy to analyze slotted systems because the number of other interferers during a slot remains unchanged. An unslotted system is more robust than a slotted system since no coordination among the users is required. However, the performance analysis is much harder because the interference level varies during the reception of a packet. In fact, it varies a bit as shown in Fig. 1. The exact solution is very difficult to obtain. These difficulties stem from two causes. First, as mentioned above, the interference level is different at each bit. We must enumerate all possible states (i.e., the number of interferers in each chip). As the number of interferers and the number of bits of a packet increase, the number of states grows to an intractable value. Second, since the code sequence (i.e., signature) pattern repeats itself in every bit, the bits of a packet are strongly correlated. Even if we can enumerate all possible states, we still cannot get around this dependence problem.

To overcome the above difficulties, we present two approximation methods to evaluate the system performance. These two approximations reduce the original problem which requires finding the correlated bit-error probabilities to the

Paper approved by I. Chlamtac, the Editor for Computer Networks of the IEEE Communications Society. Manuscript received May 15, 1992; revised January 15, 1995 and July 15, 1996. This work was supported in part by NSF Grant NCR-9318497. This paper is part of a dissertation submitted by C.$\mathrm{S}$. Hsu to the Department of Electrical Engineering, University of Southern California, Los Angeles, in partial fulfillment of the requirements for the $\mathrm{Ph}$.D. degree. This paper was presented in part at IEEE INFOCOM'93, San Francisco, CA, April 1993.

C.-S. Hsu is with the Chung-Shan Institute of Science and Technology, Lung-Tan, Taiwan, R.O.C.

V. O. K. Li is with the Communication Sciences Institute, Department of Electrical Engineering, University of Southern California, Los Angeles, CA 90089-2565 USA.

Publisher Item Identifier S 0090-6778(97)05739-5. final problem which only requires computing the bit-error probability for a single bit.

The rest of this paper is organized as follows. In Section II, we present some assumptions. In Section III, the performance analysis of a distributed unslotted hybrid frequency-hop/timehop/on-off keying (FH/TH/OOK) fiber-optic CDMA packet network is presented. We consider the throughput of the network for different packet lengths and number of frequency slots. We assume that a transmitter can always find an idle receiver waiting to receive this packet (i.e., nonpaired off) [1]. In this situation, the number of successful transmissions in the network can be greater than one in a packet duration. In Section IV, some numerical results and a summary are presented. We conclude in Section V.

\section{SYSTEM ASSUMPTIONS}

The goal of our research is to analyze the performance of CDMA packet networks using code sequences with given orthogonality properties. There are several code sequences designed for use in fiber-optic CDMA [2]-[6]. We only consider $(c, d, 1)$ code sequences. The orthogonality properties of these code sequences allow us to extract useful information which is relevant to our analysis.

The format of transmitted signals in typical fiber-optic CDMA communication networks is on-off keying (OOK) [5], [6]. In the OOK format, a " 1 " bit is transmitted as a pulse signature pattern and a " 0 " bit is transmitted as an empty bit (i.e., no pulse signature pattern is transmitted). Each bit of a packet is encoded by a specific signature pattern (we call it the timehopped $(\mathrm{TH})$ pattern in the rest of the paper) which is only one bit long. Therefore, the interference due to other simultaneous transmissions is highly correlated in time-hop/on-off keying (TH/OOK) fiber-optic CDMA communication networks. This means that the probabilities of bit errors in a packet are also highly correlated.

We consider a fully connected distributed topology, in which a transmission by any node is heard by all nodes in the network. In the following analysis, we make the following assumptions.

1) Infinite population assumption. The number of users is sufficiently large such that, with finite total offered traffic, a user is idle with probability close to one. The offered traffic is a Poisson distribution with mean $G$ (packets/packet duration). 


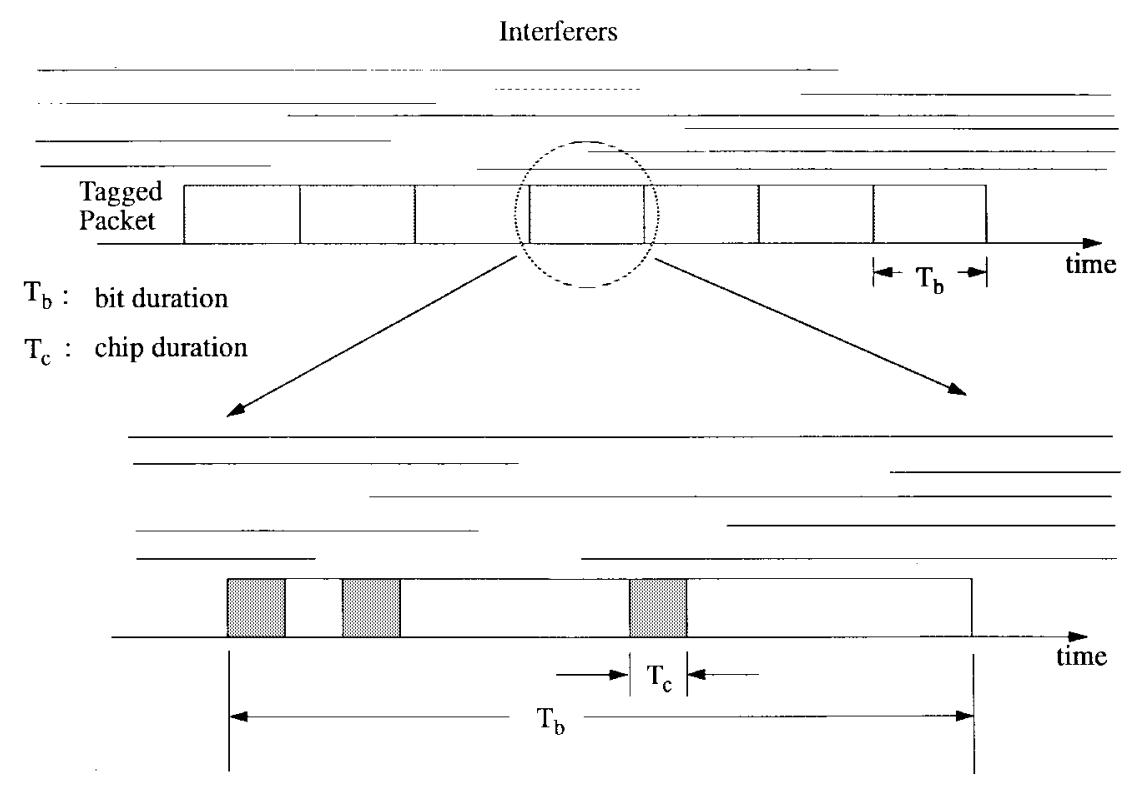

Fig. 1. Interferers overlap with the tagged packet.

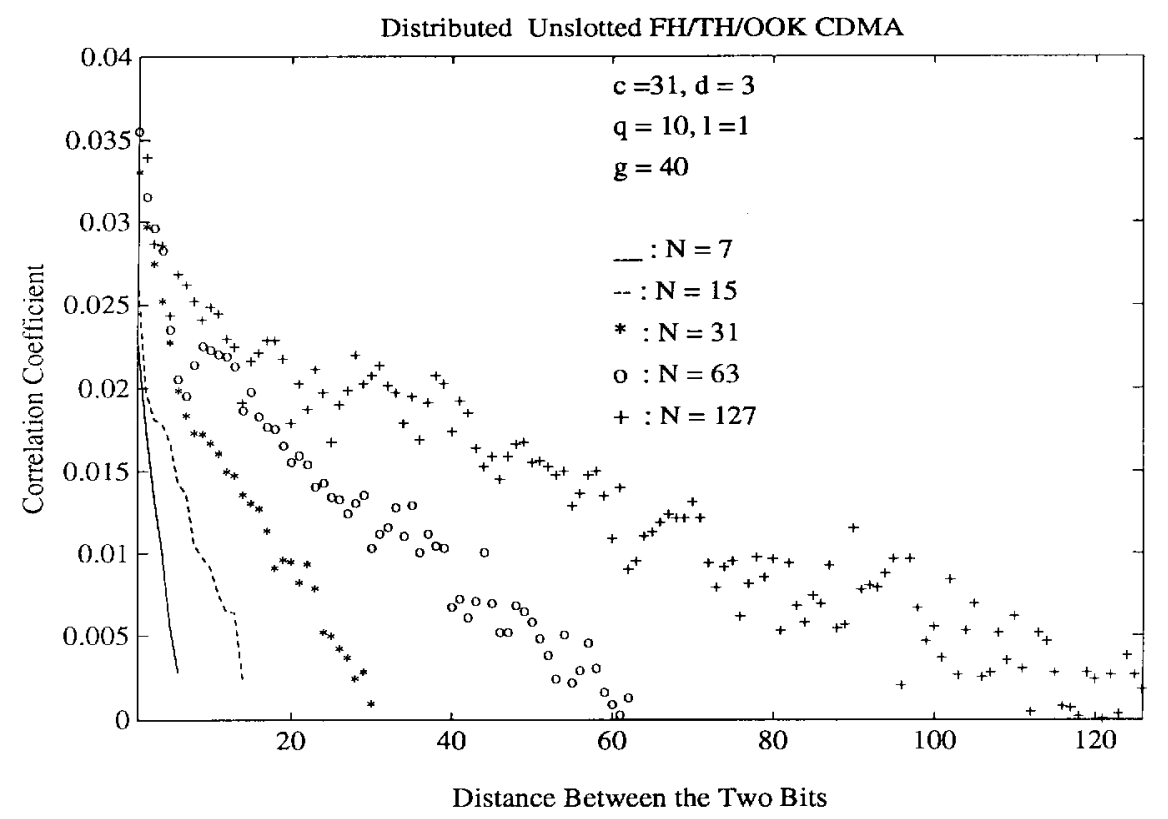

Fig. 2. Correlation coeff. of bit errors versus distance (FH/TH/OOK, $q=10$ ).

2) There are $l$ different groups which correspond to $l$ different code sequences used in the network. Each user can come from one of these $l$ groups.

3) Each packet consists of $N$ bits, and takes $T$ seconds to transmit. Each bit is divided into $c$ chips (length of signature pattern), and the number of pulses (weight of signature pattern) in each bit is $d$.

4) Each node transmits with the same intensity. Without loss of generality, assume that this intensity is unity.

5) A transmission is received with equal light power by all other nodes. Since the loss of the optical fiber is very small, this assumption holds for high-speed local-area network applications.

6) An idle receiver will capture the first packet addressed to it. The capture of this packet is successful only if any other packets are received at least one chip behind the first packet, and any other transmissions during this reception are treated as interference.

7) The analysis presented does not account for thermal noise. Instead, we are primarily concerned with multiple-access interference due to other simultaneous transmissions.

8) A separate and error-free channel is used for acknowledgment. Since the acknowledgment is very short (a few bits), we can implement a very powerful forward errorcorrecting code to achieve error-free transmission with minimal additional bandwidth.

9) The timing information needed for synchronization is perfect.

10) For simplicity of analysis, the network is assumed to be chip synchronized, namely, the time axis is divided 
into chips, and each packet can only be initiated at chip boundaries. With this assumption, our results will be pessimistic [5], [6].

\section{THROUGHPUT ANALYSIS FOR DISTRIBUTED UNSLOTTED FIBER-OPTIC FH/TH/OOK CDMA PACKET NETWORKS}

In this section, we employ a hybrid scheme $\mathrm{FH} / \mathrm{TH} / \mathrm{OOK}^{1}$ which combines frequency-hop (FH) CDMA and time-hop (TH) CDMA. Each packet is encoded by a two-layered encoding process. The inner layer encoding, which is the same as $\mathrm{TH} / \mathrm{OOK}$, uses a $\mathrm{TH}$ signature pattern (inner signature) to encode bits. FH signature patterns (outer signature) are used in the outer layer encoding process. We assume that $q$ frequency slots can be chosen. The optical signal from a transmitter is hopped from slot to slot by changing the frequency at certain instants in time called hop epochs. The intervals of time between two consecutive hop epochs are called hop intervals. We consider only fixed-rate hopping, so all intervals are of the same length $T_{\text {hop }}$. Furthermore, we assume that the hop interval $T_{\text {hop }}$ is equal to the bit interval $T_{b}$. Besides, we assume that random frequency-hopping patterns are used. If a user wants to transmit a packet, then in each hop interval, he randomly and independently chooses one of the $q$ available frequency slots to transmit.

The tagged packet method is used, namely, we arbitrarily pick a packet as our target packet, and then we evaluate the probability that the tagged packet is correctly received given that other overlapping interferers transmit during the transmission of the tagged packet. In order to find bit-error probabilities of the tagged packet, we assume that an interfering bit arrives at an instant uniformly distributed within the tagged bit. Therefore, the throughput $S$ of the system is given by

$$
S=G \cdot \operatorname{Prob}\{\text { the tagged packet is correctly received }\} .
$$

Thus, in the following sections, we will find the probability that the tagged packet is correctly received.

\section{A. Approximation Method 1 (Poisson Approximation)}

In this section, we want to find the conditions under which the number of errors in a packet can be approximated by a Poisson distribution. As mentioned in the previous section, the bit errors of a packet are strongly dependent under TH/OOK systems. However, the randomizing effect of frequency hopping $(\mathrm{FH})$ will reduce this dependency as $q$ (i.e., the number of frequency slots which corresponds to the processing gain in the $\mathrm{FH}$ scheme) increases.

The correlation coefficient is a measure of the correlation of two random variables. Define $X_{\alpha}$ as an error indicator random variable:

$$
X_{\alpha}=\left\{\begin{array}{ll}
1, & \text { if bit } \alpha \text { is incorrect } \\
0, & \text { otherwise }
\end{array} \quad \forall \alpha \in I\right.
$$

where $I=\{1,2, \cdots, N\}$, and $N$ is the number of bits per packet. Given $\alpha, \beta \in I$, and that the distance between bits $\alpha$

\footnotetext{
${ }^{1}$ Some prefer different names, e.g., multiwavelength, multicolor CDMA.
}

and $\beta$ is $|\alpha-\beta|$, then the correlation coefficient $\rho_{\alpha \beta}$ of $X_{\alpha}$ and $X_{\beta}$ is defined as

$$
\rho_{\alpha \beta}(|\alpha-\beta|) \equiv \frac{E\left[\left(X_{\alpha}-E\left(X_{\alpha}\right)\right)\left(X_{\beta}-E\left(X_{\beta}\right)\right)\right]}{\sigma_{\alpha} \sigma_{\beta}}
$$

where $\sigma_{i}^{2}=E\left[\left(X_{i}-E\left(X_{i}\right)^{2}\right], i=\alpha, \beta\right.$.

Since the exact solution for the correlation coefficient is not easy to obtain, simulation results are shown instead. The Monte Carlo method is used in the simulation where the simulation time is 1000000 packet times. For $q=10$, we plot the correlation coefficient of bit errors versus the distance between them in Fig. 2 for different $N$ with $c=31$, $d=3, l=1, G=40$. The correlation coefficient decreases with increasing distance between two bits. The correlation coefficient increases as $N$ increases under the same distance. In Fig. 3, we plot the same curves with the same parameters, except that $q$ is changed from 10 to 30. As expected, the correlation coefficient decreases as $q$ increases. Furthermore, the correlation coefficient decreases as the number of groups $l$ (i.e., number of code sequences) increases (Fig. 4). Because the probability of bit error is dominated by the interference from the same group, this dominance decreases as the number of groups increases. Unfortunately, the upper bound for the correlation coefficient is not easy to obtain. This bound should be a function of $q, l, c$, and $d$.

For comparison, the correlation coefficients of bit errors for TH/OOK systems are shown in Fig. 5 for different $N$ with $c=31, d=3, N=31, G=10, l=1$. The correlation coefficients of $\mathrm{TH} / \mathrm{OOK}$ systems are much larger than those of $\mathrm{FH} / \mathrm{TH} / \mathrm{OOK}$ systems. This indicates that the Poisson approximation cannot be applied to TH/OOK systems. From the above observation, the correlation coefficients of bit errors between two bits decreases as $q$ increases. Uncorrelatedness does not imply independence. However, the above fact does indicate that the Poisson approximation is suitable as $q$ increases.

1) Bound for Difference Between Actual Distribution and Poisson Distribution: The bound for the difference between the actual distribution and the Poisson distribution is derived by Chen [7], and is modified to a more usable form by Arratia et al. [8]. Let $I$ be an arbitrary index set, and for $\alpha \in I$, let $X_{\alpha}$ be a Bernoulli random variable with $P\left(X_{\alpha}=\right.$ $1)=1-P\left(X_{\alpha}=0\right)=p_{\alpha}$. Let $W \equiv \Sigma_{\alpha \in I} X_{\alpha}$ and $\lambda \equiv E\{W\}=\Sigma_{\alpha \in I} p_{\alpha}, \lambda \in(0, \infty)$. For each $\alpha \in I$, suppose we have chosen $B_{\alpha} \subset I$ with $\alpha \in B_{\alpha}$; then $B_{\alpha}$ can be thought of as a neighborhood of dependence for $\alpha$, such that $X_{\alpha}$ is independent or nearly independent of all of the $X_{\beta}$ for $\beta \notin B_{\alpha}$.

Define three parameters as follows [8]:

$$
\begin{aligned}
b_{1} & \equiv \sum_{\alpha \in I} \sum_{\beta \in B_{\alpha}} p_{\alpha} \cdot p_{\beta} \\
b_{2} & \equiv \sum_{\alpha \in I} \sum_{\alpha \neq \beta \in B_{\alpha}} E\left\{X_{\alpha} X_{\beta}\right\} \\
b_{3}^{\prime} & \equiv \sum_{\alpha \in I} E\left|E\left\{X_{\alpha}-p_{\alpha} \mid \sum_{\beta \in I-B_{\alpha}} X_{\beta}\right\}\right| .
\end{aligned}
$$




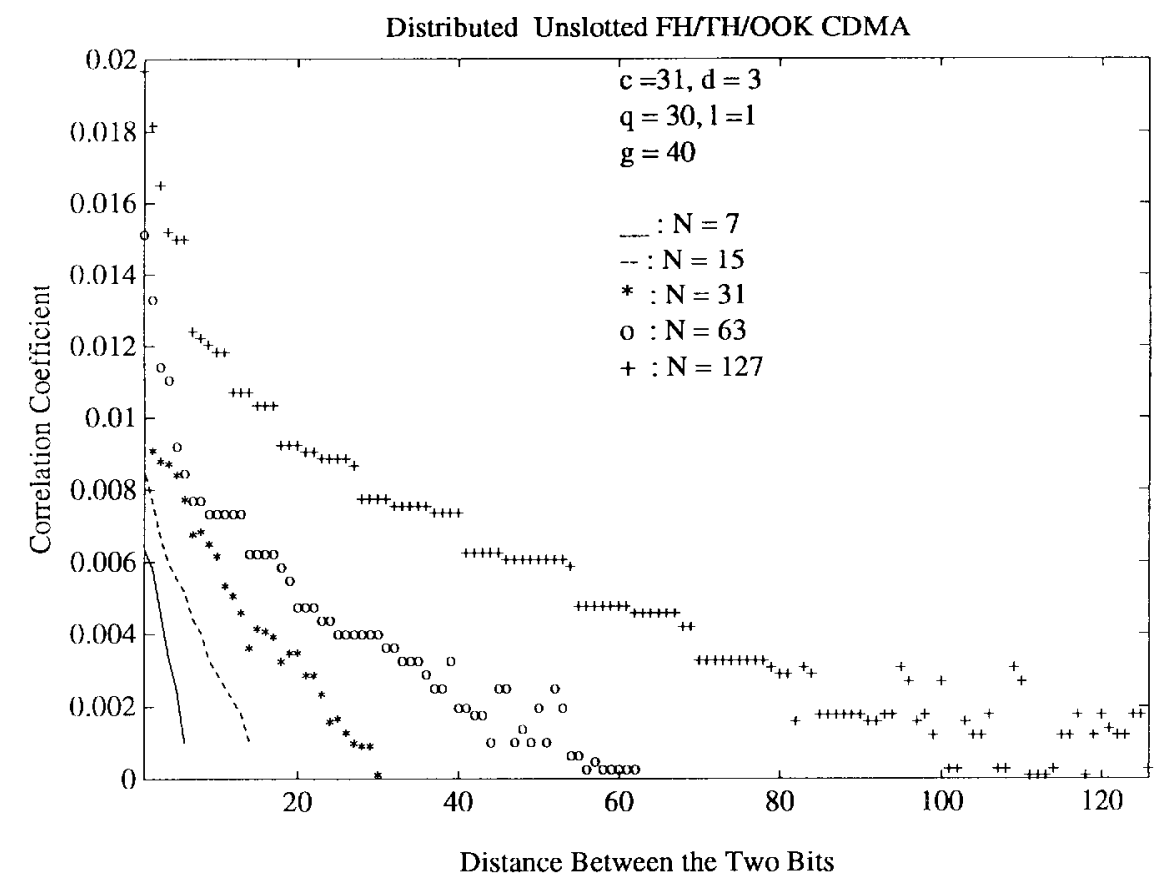

Fig. 3. Correlation coeff. of bit errors versus distance (FH/TH/OOK, $q=30$ ).

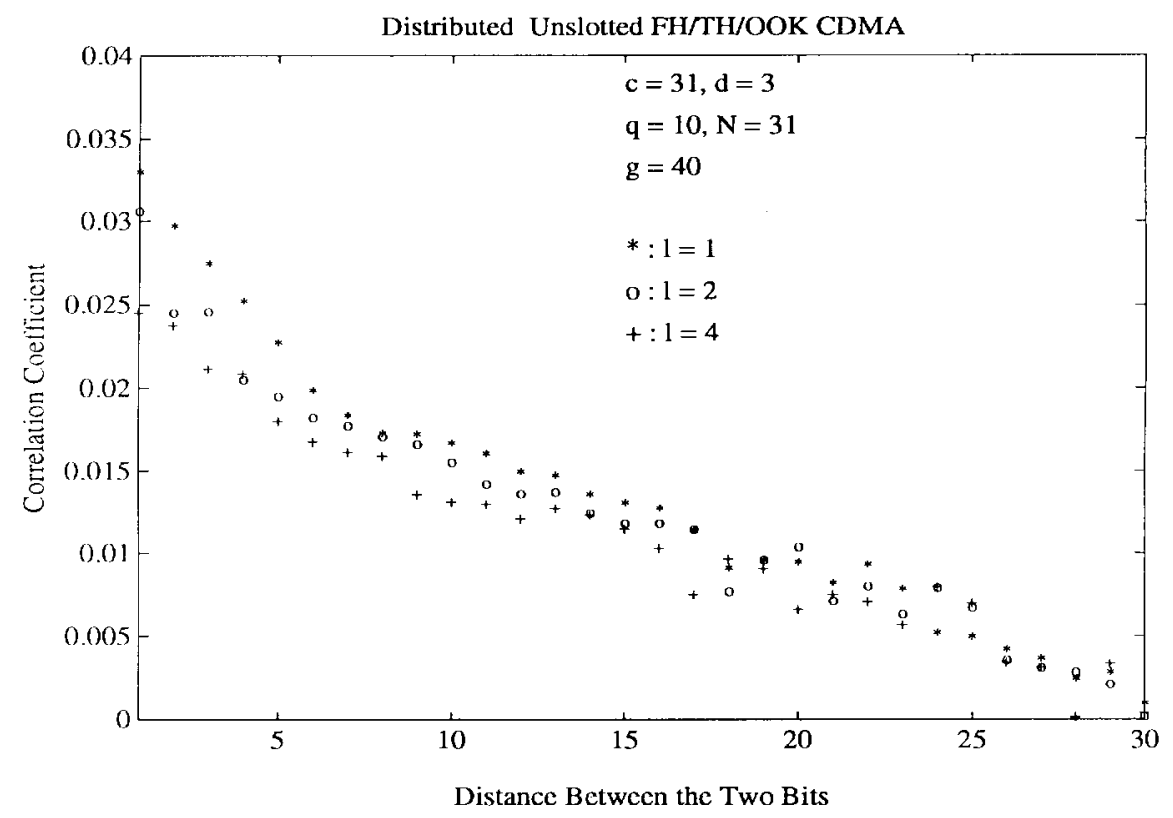

Fig. 4. Correlation coeff. of bit errors versus distance for $l=1,2,4$ (FH/TH/OOK).

Quoting from [8]: "Loosely, $b_{1}$ measures the neighborhood size, $b_{2}$ measures the expected number of neighbors of a given occurrence and $b_{3}^{\prime}$ measures the dependence between an event and the number of occurrences outside its neighborhood."

Let $Y$ be a Poisson random variable with mean $\lambda . P(Y=$ $k)=\left(e^{-\lambda} \lambda^{k} / k !\right), k=0,1,2, \cdots$. The total variation distance (TVD), which is a measure of the difference between the actual distribution $W$ and the Poisson distribution $Y$, is given by [8]

$$
\begin{aligned}
& 2 \limsup _{A \subset Z^{+}}|P(W \in A)-P(Z \in A)| \\
& \quad \leq 2\left[\left(b_{1}+b_{2}\right) \frac{1-e^{-\lambda}}{\lambda}+b_{3}^{\prime} \cdot \min \left(1,1.4 \lambda^{2}\right)\right]
\end{aligned}
$$

where $Z^{+} \equiv\{0,1,2, \cdots\}$.
By choosing a neighborhood of size one, i.e., $B_{\alpha}=\alpha$,

$$
\begin{aligned}
& b_{1}=\sum_{\alpha \in I} \sum_{\beta \in B_{\alpha}} p_{\alpha} \cdot p_{\beta}=N p_{\alpha}^{2}=\frac{\lambda^{2}}{N} \\
& b_{2}=\sum_{\alpha \in I} \sum_{\alpha \neq \beta \in B_{\alpha}} E\left\{X_{\alpha} X_{\beta}\right\}=0 .
\end{aligned}
$$

In the asymptotic case, i.e., $q \rightarrow \infty$, following a similar procedure in [9], the error contribution due to $b_{3}$ can be neglected. Therefore, the total variation distance can be bounded by $(\lambda / N)$ as $q \rightarrow \infty$.

2) Derivation of $p_{\alpha}$ and $\lambda$ : The analysis uses the tagged packet technique to compute the probability that the tagged packet is correctly received while other interferers are trans- 


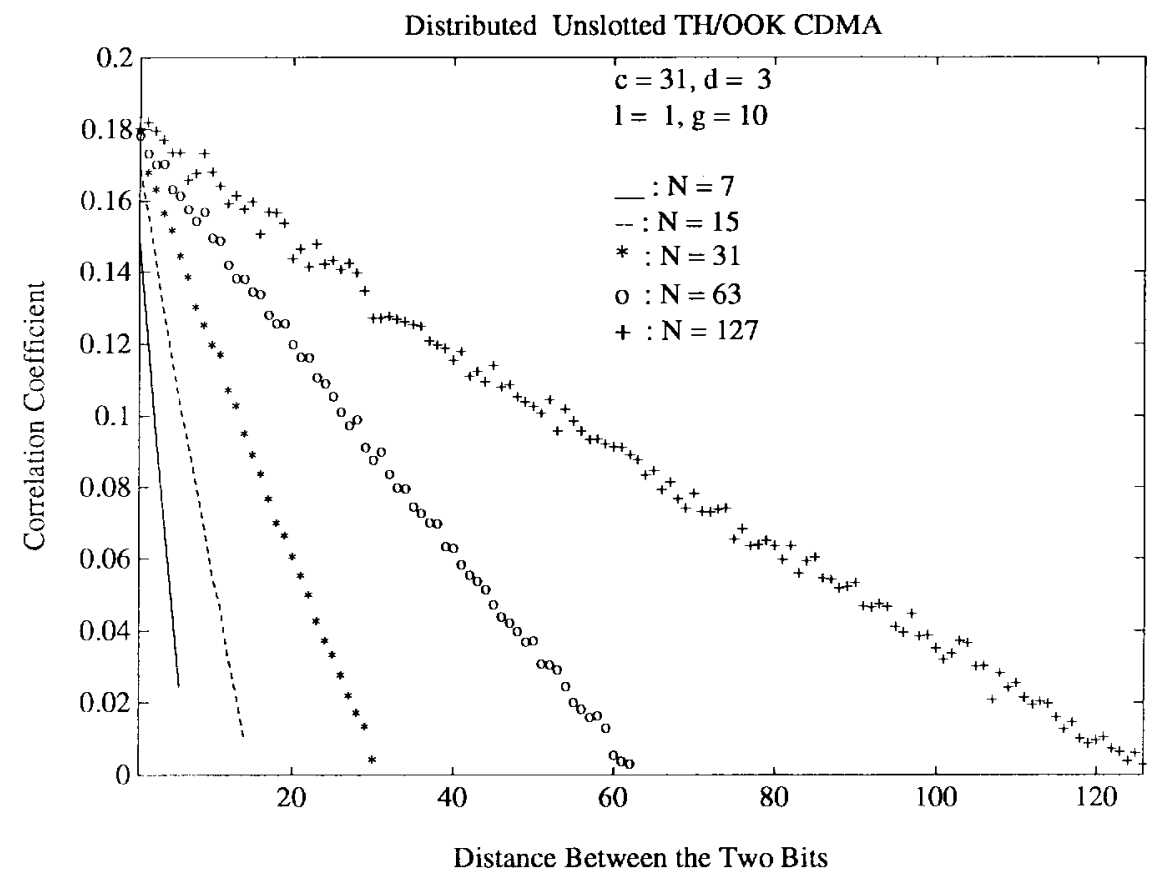

Fig. 5. Correlation coeff. of bit errors versus distance (TH/OOK).

mitting during the transmission of the tagged packet. The error probability of bit $\alpha$ in the tagged packet is $p_{\alpha}$, i.e., $P\left(X_{\alpha}=1\right)$. We assume that there are $l$ groups of code sequences (i.e., signatures) used in the system.

$$
\begin{aligned}
p_{\alpha} & =P\left(X_{\alpha}=1\right) \\
& =\sum_{u=1}^{l} P\left(X_{\alpha}=1 \mid \text { the tagged packet uses signature } u\right)
\end{aligned}
$$$$
\text { - } P \text { (the tagged packet uses signature } u \text { ). }
$$

For simplicity, we assume that a packet is transmitted using one of $l$ signatures with equal probability, i.e., $P$ (the tagged packet uses signature $u)=1 / l$. Since each signature has the same orthogonality property, $P\left(X_{\alpha}=1 \mid\right.$ the tagged packet uses signature $u$ ) is independent of $u$. Without loss of generality, we assume that the tagged packet uses signature pattern 1, i.e., $u=1$. Let $E$ be the event that the tagged packet uses signature pattern 1 . Therefore,

$$
p_{\alpha}=P\left(X_{\alpha}=1\right)=P\left(X_{\alpha}=1 \mid E\right) .
$$

Assume that bit $\alpha$ is a " 0 " or " 1 " bit with probability $1 / 2$. Let $Z$ be the event that bit $\alpha$ is a " 0 " bit. Since bit error can occur only when bit $\alpha$ is a " 0 " bit, $P\left(X_{\alpha}=1 \mid E\right)=\frac{1}{2} P\left(X_{\alpha}=\right.$ $1 \mid Z, E)$.

Since the offered traffic is Poisson with average rate $G$, the offered traffic due to interferers arriving during the tagged bit $\alpha$ is also Poisson with average rate $G \cdot(N+1 / N)$, where $N$ is the number of bits per packet. Thus,

$$
P\left(X_{\alpha}=1 \mid Z, E\right)=\sum_{i=0}^{\infty} P\left(X_{\alpha}=1 \mid i, Z, E\right) \cdot P(i \mid Z, E)
$$

where $P(i \mid Z, E)=\left(e^{-\hat{G}} \hat{G}^{i} / i !\right), \hat{G}=G \cdot(N+1 / N)$.

For conciseness, we use the following shorthand notation to represent conditional probabilities. Let $P\left(X_{\alpha}=1 \mid i, Z, E\right)$ represent the error probability of bit $\alpha$, given $i$ other interferers in the tagged bit $\alpha$, tagged bit $\alpha$ is a " 0 " bit, and the tagged packet uses signature 1 .

An interferer can hit the tagged bit $\alpha$ if the bit of the interferer overlapping the tagged bit has the same frequency as the tagged bit, and this interfering bit is a " 1 " bit. We assume that the frequency is randomly chosen by a bit from one of $q$ frequency slots with probability $1 / q$. Since the frequency of a bit and the bit being a " 0 " or " 1 " bit are independent, the probability for an interferer to hit the tagged bit is $1 / 2 q$. Let $j$ be the number of interferers that can hit the tagged bit $\alpha$. Therefore, we obtain

$$
\begin{aligned}
P\left(X_{\alpha}\right. & =1 \mid i, Z, E) \\
& =\sum_{j=0}^{\infty} P\left(X_{\alpha}=1 \mid j, i, Z, E\right) \cdot P(j \mid i, Z, E)
\end{aligned}
$$

where $P(j \mid i, Z, E)=B(i, j,(1 / 2 q))$ and $B(n, k, p) \equiv$ $\left(\begin{array}{c}n \\ k\end{array}\right) p^{k}(1-p)^{n-k}$ is the probability mass function of the binomial distribution with parameters $n, k, p$. Since we assume that interferers can come from one of $l$ groups with equal probability, we have

$$
\begin{aligned}
P\left(X_{\alpha}\right. & =1 \mid j, i, Z, E) \\
& =\sum_{\underline{j} \in \Sigma_{j}} P\left(X_{\alpha}=1 \mid \underline{j}, j, i, Z, E\right) \cdot P(\underline{j} \mid j, i, Z, E)
\end{aligned}
$$

where

$$
\begin{aligned}
\underline{j} & \equiv\left(j_{1}, j_{2}, \cdots, j_{l}\right) \\
j_{k} & \equiv \text { number of interferers from group } k \\
\Sigma_{j} & \equiv\left\{\underline{j} \mid \sum_{k=1}^{l} j_{k}=j\right\}
\end{aligned}
$$

$$
P(\underline{j} \mid j, i, Z, E)=\left(\begin{array}{c}
j \\
j_{1}, j_{2}, \cdots, j_{l}
\end{array}\right)\left(\frac{1}{l}\right)^{j}
$$


From the orthogonality property of the signatures, interferers from different groups can overlap the tagged signature at only one chip position. However, severe damage occurs when the interferers come from the same group as the tagged signature. If these interferers initiate at the first chip position of the tagged signature, then they will overlap all of the $d$ chip positions of the tagged signature. Let $B$ be the event that an interferer initiates at the first chip position of the tagged signature. Obviously, $P(B)=(1 / c)$. We observe that $P\left(X_{\alpha}=1 \mid \underline{j}, j, i, Z, E\right)=P\left(X_{\alpha}=1 \mid \underline{j}, Z, E\right)$ because $\underline{j}$ provides all of the information needed to compute the bit-error probability.

Let $H$ be the event that at least one interferer is from the first group (i.e., the same group as the tagged packet). The probability of $H$ is given by $1-(1-(1 / c))^{j_{1}}$. Therefore, we have

$$
\begin{aligned}
& P\left(X_{\alpha}=1 \mid \underline{j}, Z, E\right) \\
& =P\left(X_{\alpha}=1 \mid H, \underline{j}, Z, E\right) \cdot P(H \mid \underline{j}, Z, E) \\
& \quad+P\left(X_{\alpha}=1 \mid \bar{H}, \underline{j}, Z, E\right) \cdot P(\bar{H} \mid \underline{j}, Z, E)
\end{aligned}
$$

where

$$
\begin{aligned}
& P(H \mid \underline{j}, Z, E)=1-\left(1-\frac{1}{c}\right)^{j_{1}} \\
& P(\bar{H} \mid \underline{j}, Z, E)=\left(1-\frac{1}{c}\right)^{j_{1}}
\end{aligned}
$$

$\bar{H}$ is the complement of $H$.

Since the threshold of the threshold detector is set to $d$, we obtain

$$
\begin{aligned}
& P\left(X_{\alpha}=1 \mid H, \underline{j}, Z, E\right)=1 \cdot U\left(j_{1}-1\right) \\
& P\left(X_{\alpha}=1 \mid \bar{H}, \underline{j}, Z, E\right)=P\left(I_{t} \geq d \mid \bar{H}, \underline{j}, Z, E\right)
\end{aligned}
$$

where $U(t)$ is the step function of $t$ and $I_{t}$ is the accumulated interference level at the input of the threshold detector.

Let $\xi_{k}$ be the accumulated interference level at the input of the threshold detector due to the interferers from group $k$, where $k \in\{1,2, \cdots, l\}$; then $I_{t}=\Sigma_{k=1}^{l} \xi_{k}$. Therefore, we have

$$
P\left(\xi_{k} \mid \bar{H}, \underline{j}, Z, E\right)=B\left(j_{k}, \xi_{k}, P_{v k}\right)
$$

where

$$
P_{v k}= \begin{cases}\frac{d(d-1)}{c-1}, & \text { if } k=1 \\ \frac{d^{2}}{c}, & \text { if } k \neq 1 .\end{cases}
$$

Since $\left\{\xi_{k}, k=1,2, \cdots, l\right\}$ are independent random variables, we have

$$
\begin{aligned}
P\left(I_{t} \mid \bar{H}, \underline{j}, Z, E\right) & \\
= & P\left(\xi_{1} \mid \bar{H}, \underline{j}, Z, E\right) * P\left(\xi_{2} \mid \bar{H}, \underline{j}, Z, E\right) \\
& * \cdots * P\left(\xi_{l} \mid \bar{H}, \underline{j}, Z, E\right)
\end{aligned}
$$

where $*$ denotes the convolution operator.
Because the number of errors in a packet $W=\sum_{\beta}^{N} X_{\beta}$, and $X_{1}, X_{2}, \cdots, X_{N}$ have the same probability distribution, we have

$$
\lambda=E\{W\}=\sum_{\beta=1}^{N} E\left\{X_{\beta}\right\}=N \cdot p_{\alpha}
$$

where $\alpha$ is an arbitrary bit of a packet.

Now, we can use a Poisson distribution for a random variable $Y$ with the same mean $\lambda$ to approximate the original distribution of $W$, which is the sum of $N$ dependent random variables. Since no error-correcting codes are employed, the probability that the tagged packet is correctly received is given by $e^{-\lambda}$.

\section{B. Approximation Method 2}

In the first approximation method, we assume that the number of interferers in each bit is the same. Actually, given the number of interferers for a tagged packet, the number of interferers in each bit is different. Furthermore, some interferers may start transmission during the tagged packet, and some may leave during the tagged packet. In this section, we will consider the distributions of these starting (or final) and leaving (or initial) interferers. This concept is based on the paper of Tarr et al. [10]. From these initial and final interferer distributions, we can find the expected value of the bit-error probability for each bit in a packet given the total number of interferers and the number of initial interferers in the tagged packet.

First, we divide $I_{T}$ total interferers transmitting during the tagged packet into $I_{i}$ initial interferers and $I_{f}$ final interferers. Define

$$
\begin{array}{r}
i_{j} \equiv\{\text { number of initial interferers in } \\
j \text { th bit of the tagged packet }\} \\
f_{j} \equiv\{\text { number of final interferers in } \\
j \text { th bit of the tagged packet }\} .
\end{array}
$$

From [10], we obtain

$$
\begin{aligned}
P\left(i_{j+1}\right. & \left.=m-k \mid i_{j}=m, I_{i}, I_{T}\right) \\
= & B\left(m, k, \frac{1}{N-j+1}\right) \\
P\left(i_{j+1}=k \mid I_{i}, I_{T}\right) & \\
= & \sum_{m=0}^{I_{i}-k} P\left(i_{j+1}=k \mid i_{j}=k+m, I_{i}, I_{T}\right) \\
& \cdot P\left(i_{j}=k+m \mid I_{i}, I_{T}\right)
\end{aligned}
$$

with initial condition $P\left(i_{1}=I_{i}\right)=1$ and

$$
\begin{aligned}
P\left(f_{j+1}=m+k \mid f_{j}=m, I_{i}, I_{T}\right) & \\
= & B\left(I_{f}-m, k, \frac{1}{N-j}\right) \\
P\left(f_{j+1}=k \mid I_{i}, I_{T}\right) & \\
= & \sum_{m=0}^{k} P\left(f_{j+1}=k \mid f_{j}=k-m, I_{i}, I_{T}\right) \\
& \cdot P\left(f_{j}=k-m \mid I_{i}, I_{T}\right)
\end{aligned}
$$

with initial condition $P\left(f_{0}=0\right)=1$. 


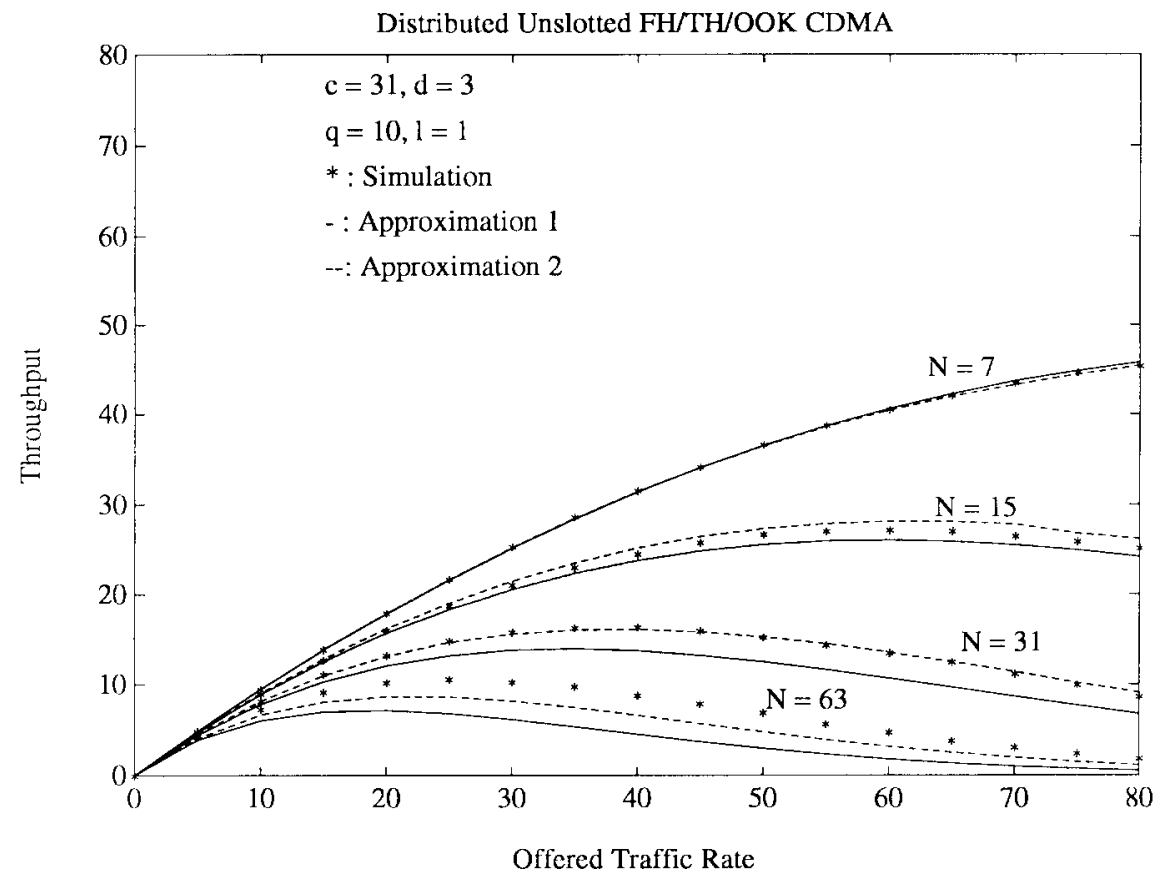

Fig. 6. Throughput versus offered traffic rate for different $N$ 's.

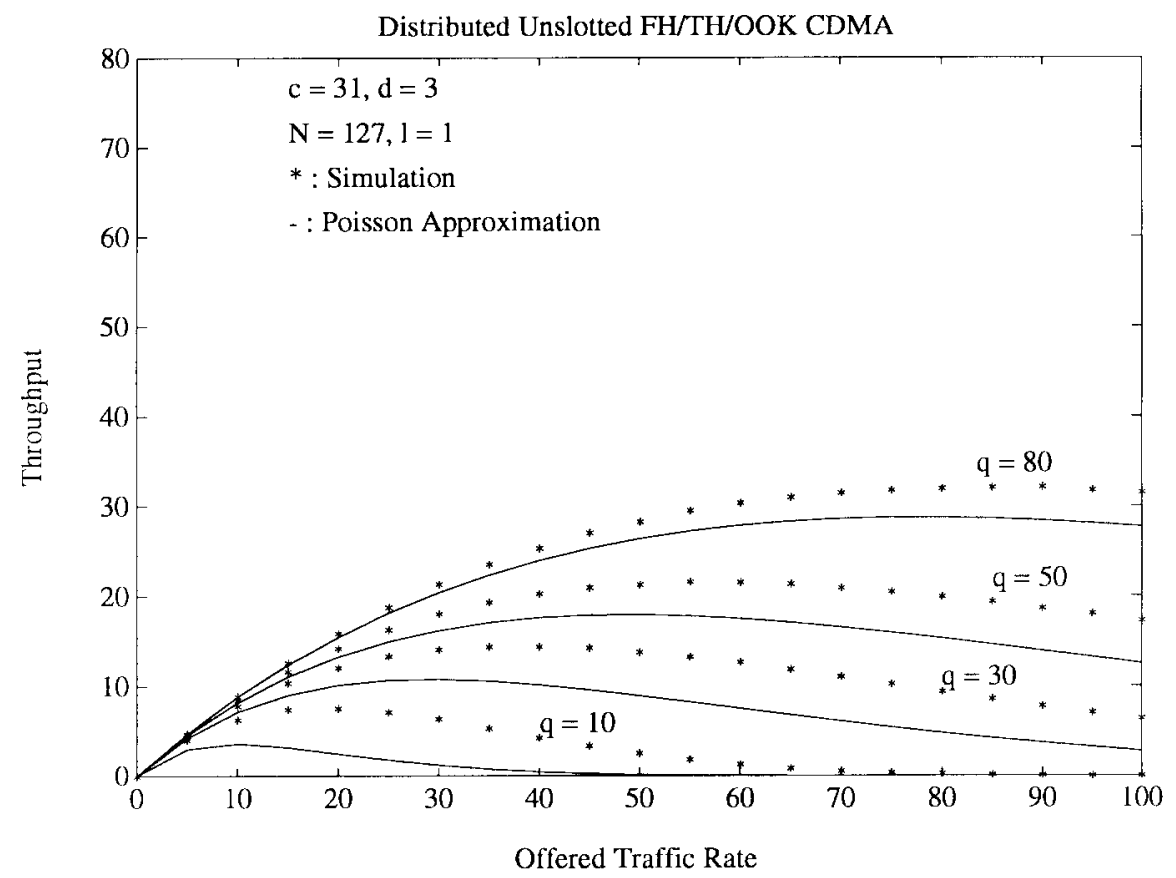

Fig. 7. Throughput versus offered traffic rate for different $q$ 's.

Given $I_{i}$ and $I_{T}, i_{j}$ and $f_{j}$ are independent, then the distribution of the total number of interferers in each bit of the tagged packet is given by

$$
\begin{aligned}
& P\left(t_{j}=k \mid I_{i}, I_{T}\right) \\
& \quad=\sum_{m=0}^{k} P\left(i_{j}=m \mid I_{i}, I_{T}\right) \cdot P\left(f_{j}=k-m \mid I_{i}, I_{T}\right) .
\end{aligned}
$$

Therefore, the expected values of bit-error probabilities for each bit $j$ of the tagged packet given $I_{i}$ and $I_{T}$ are

$$
\begin{aligned}
& P\left(X_{j}=1 \mid I_{i}, I_{T}\right) \\
&= \sum_{m=0}^{I_{T}} P\left(X_{j}=1 \mid m, I_{i}, I_{T}\right) \cdot P\left(t_{j}=m \mid I_{i}, I_{T}\right) \\
& \quad j=1,2, \cdots, N .
\end{aligned}
$$

We assume that an interferer is either an initial interferer or a final interferer with equal probability. Besides, to simplify the 
TABLE I

Simulation Versus Approx. Methods for $c=31, d=3, q=, l=1$

\begin{tabular}{|c|c|c|c|c|c|}
\hline \multirow{2}{*}{$\mathrm{N}$} & \multirow{2}{*}{$\begin{array}{l}\text { Offered } \\
\text { Traffic } \\
\text { Rate }\end{array}$} & \multirow{2}{*}{$\begin{array}{c}\text { Total } \\
\text { Variation } \\
\text { Distance }\end{array}$} & \multicolumn{3}{|c|}{ Throughput } \\
\hline & & & Simulation & Approx. 1 & Approx. 2 \\
\hline \multirow{4}{*}{7} & 10 & 0.007116 & 9.46307 & 9.4509 & 9.4576 \\
\hline & 20 & 0.010710 & 17.8795 & 17.834 & 17.859 \\
\hline & 40 & 0.011182 & 31.5429 & 31.391 & 31.438 \\
\hline & 80 & 0.015944 & 45.3963 & 45.795 & 45.424 \\
\hline \multirow{4}{*}{15} & 10 & 0.038326 & 8.96680 & 8.8601 & 9.0037 \\
\hline & 20 & 0.059147 & 15.9999 & 15.644 & 16.136 \\
\hline & 40 & 0.065284 & 24.5253 & 23.796 & 25.177 \\
\hline & 80 & 0.031830 & 25.1205 & 24.207 & 26.151 \\
\hline \multirow{4}{*}{31} & 10 & 0.132706 & 8.19793 & 7.7871 & 8.1273 \\
\hline & 20 & 0.176125 & 13.2019 & 12.038 & 13.087 \\
\hline & 40 & 0.187122 & 16.2987 & 13.675 & 16.053 \\
\hline & 80 & 0.099751 & 8.67253 & 6.7635 & 9.1075 \\
\hline \multirow{4}{*}{63} & 10 & 0.326620 & 7.22800 & 6.0152 & 6.6314 \\
\hline & 20 & 0.416490 & 10.1618 & 7.1283 & 8.6366 \\
\hline & 40 & 0.330076 & 8.78667 & 4.5157 & 6.5918 \\
\hline & 80 & 0.230794 & 1.88533 & 0.5280 & 1.1435 \\
\hline \multirow{4}{*}{127} & 10 & 0.708152 & 6.29087 & 3.5892 & 4.4491 \\
\hline & 20 & 0.703230 & 7.57373 & 2.4994 & 3.8277 \\
\hline & 40 & 0.506468 & 4.34213 & 0.4924 & 1.1667 \\
\hline & 80 & 0.405522 & 0.25173 & 0.0032 & 0.0211 \\
\hline
\end{tabular}

analysis, we incorrectly assume that the bit errors among the tagged packet are independent. Since no error-correcting codes are used, the probability that the tagged packet is correctly received is given by

$P$ (the tagged packet is correctly received)

$$
\begin{aligned}
= & \sum_{I_{T}=0}^{\infty} \sum_{I_{i}=0}^{I_{T}} \prod_{j=1}^{N}\left(1-P\left(X_{j}=1 \mid I_{i}, I_{T}\right)\right. \\
& \left.\cdot P\left(I_{i} \mid I_{T}\right)\right) \cdot P\left(I_{T}\right)
\end{aligned}
$$

where

$$
\begin{aligned}
P\left(I_{i} \mid I_{T}\right) & =B\left(I_{T}, I_{i}, \frac{1}{2}\right) \\
P\left(I_{T}\right) & =\frac{e^{-2 G}(2 G)^{I_{T}}}{I_{T} !} \quad I_{T}=0,1,2, \cdots .
\end{aligned}
$$

The above " $2 G$ " is due to the Poisson distributions for the number of initial and final interferers in the tagged packet, each with mean $G$. We can follow the same technique as in the previous section to derive $P\left(X_{j}=1 \mid I_{i}, I_{T}\right)$, where $j=1,2, \cdots, N$.

\section{NUMERICAL EXAMPLES AND SUMMARY}

In this section, we discuss some numerical results on the accuracy of these two approximation methods and the throughput performance of unslotted CDMA networks. Since the exact solution is not available, simulation is performed

\begin{tabular}{|c|c|c|c|c|c|}
\hline \multirow{2}{*}{$\mathrm{N}$} & \multirow{2}{*}{$\begin{array}{l}\text { Offered } \\
\text { Traffic } \\
\text { Rate }\end{array}$} & \multirow{2}{*}{$\begin{array}{c}\text { Total } \\
\text { Variation } \\
\text { Distance }\end{array}$} & \multicolumn{3}{|c|}{ Throughput } \\
\hline & & & Simulation & Approx. 1 & Approx. 2 \\
\hline \multirow{4}{*}{7} & 10 & 0.004654 & 9.72903 & 9.7137 & 9.7051 \\
\hline & 20 & 0.007358 & 18.8225 & 18.783 & 18.748 \\
\hline & 40 & 0.007408 & 34.2757 & 34.231 & 34.046 \\
\hline & 80 & 0.008014 & 50.1699 & 50.369 & 49.648 \\
\hline \multirow{4}{*}{15} & 10 & 0.022583 & 9.45553 & 9.3966 & 9.4685 \\
\hline & 20 & 0.038316 & 17.6970 & 17.483 & 17.738 \\
\hline & 40 & 0.051789 & 29.2641 & 28.650 & 29.424 \\
\hline & 80 & 0.026816 & 30.2165 & 29.685 & 31.029 \\
\hline \multirow{4}{*}{31} & 10 & 0.079819 & 9.02083 & 8.7930 & 8.9789 \\
\hline & 20 & 0.126407 & 15.9096 & 15.146 & 15.787 \\
\hline & 40 & 0.150857 & 22.1848 & 20.069 & 21.890 \\
\hline & 80 & 0.098028 & 12.5579 & 10.310 & 12.877 \\
\hline \multirow{4}{*}{63} & 10 & 0.225209 & 8.44247 & 7.6997 & 8.0767 \\
\hline & 20 & 0.316332 & 13.6723 & 11.368 & 12.521 \\
\hline & 40 & 0.320163 & 14.2819 & 9.8476 & 12.206 \\
\hline & 80 & 0.215358 & 3.12267 & 1.2438 & 2.3110 \\
\hline \multirow{4}{*}{127} & 10 & 0.521243 & 7.82963 & 5.9040 & 6.5497 \\
\hline & 20 & 0.658456 & 11.3698 & 6.4036 & 7.9312 \\
\hline & 40 & 0.491185 & 8.02013 & 2.3711 & 3.9242 \\
\hline & 80 & 0.376431 & 0.44586 & 0.3130 & 0.0884 \\
\hline
\end{tabular}
to verify the accuracy of the approximations. Two methods
TABLE II

Simulation Versus Approx. Methods For $c=31, d=3, q=10, l=2$

are used to measure the accuracy. In the first method, we measure the total variation distance between approximation 1 (i.e., Poisson approximation) and simulation results on the distribution of the number of errors in a packet. In the second method, we measure the differences in throughput between the simulation results and that due to approximations 1 and 2 .

Fig. 6 shows the throughput versus offered traffic rate for different $N$ 's, with parameters $c=31, d=3, q=10, l=1$. For each $N$, the results of approximation 2 are closer to the simulation results than those of approximation 1 . However, the approximation and the simulation results deviate very little as $N$ increases. Table I shows the total variation distance between approximation 1 and the simulation. Besides, they also show the throughputs for the approximations and simulation. The total variation distance increases as $N$ increases, which is not expected. The reason is not obvious. However, from Section III-A, we observe that the correlation coefficient of bit errors increases as $N$ increases for the same distance between 2 bits. This may be because the effect of $N$ dominates the effect of $\lambda$ on the total variation distance.

As $l$ (i.e., the number of signatures) increases, the approximation methods improve (cf. Tables I-III). This is expected. Since the contribution to the correlation of bit errors stems mainly from the interference of the same signature group, as the number of signature groups increases, the error contribution from the same group decreases. Approximation 2 is better than approximation 1 in most cases. This is because approximation 2 considers the distribution of the number of interferers in each bit. On the other hand, approximation 1 (Poisson 
TABLE III

Simulation Versus Approx. Methods for $c=31, d=3, q=10, l=4$

\begin{tabular}{|c|c|c|c|c|c|}
\hline \multirow{2}{*}{$\mathrm{N}$} & \multirow{2}{*}{$\begin{array}{c}\text { Offered } \\
\text { Traffic } \\
\text { Rate }\end{array}$} & \multirow{2}{*}{$\begin{array}{c}\text { Total } \\
\text { Variation } \\
\text { Distance }\end{array}$} & \multicolumn{3}{|c|}{ Throughput } \\
\hline & & & Simulation & Approx. 1 & Approx. 2 \\
\hline \multirow{4}{*}{7} & 10 & 0.002387 & 9.85123 & 9.8480 & 9.8308 \\
\hline & 20 & 0.004646 & 19.2963 & 19.273 & 19.200 \\
\hline & 40 & 0.004463 & 35.6961 & 35.678 & 35.337 \\
\hline & 80 & 0.014704 & 51.7461 & 52.327 & 50.265 \\
\hline \multirow{4}{*}{15} & 10 & 0.012563 & 9.70756 & 9.6770 & 9.6942 \\
\hline & 20 & 0.022203 & 18.5912 & 18.474 & 18.542 \\
\hline & 40 & 0.036049 & 31.6908 & 31.308 & 31.619 \\
\hline & 80 & 0.030196 & 32.8581 & 32.213 & 33.175 \\
\hline \multirow{4}{*}{31} & 10 & 0.045153 & 9.46693 & 9.3440 & 9.4379 \\
\hline & 20 & 0.077918 & 17.4486 & 16.974 & 17.317 \\
\hline & 40 & 0.101738 & 25.4938 & 24.108 & 25.326 \\
\hline & 80 & 0.093757 & 14.4706 & 12.207 & 14.767 \\
\hline \multirow{4}{*}{63} & 10 & 0.131433 & 9.11387 & 8.7119 & 8.9051 \\
\hline & 20 & 0.206560 & 15.7576 & 14.329 & 15.019 \\
\hline & 40 & 0.257081 & 17.9031 & 14.294 & 16.311 \\
\hline & 80 & 0.192544 & 3.74773 & 1.7531 & 3.0648 \\
\hline \multirow{4}{*}{127} & 10 & 0.314007 & 8.74747 & 7.5731 & 7.9679 \\
\hline & 20 & 0.474125 & 13.7320 & 10.212 & 11.403 \\
\hline & 40 & 0.413457 & 10.5177 & 5.0254 & 6.9765 \\
\hline & 80 & 0.350075 & 0.53466 & 0.0036 & 0.1592 \\
\hline
\end{tabular}

TABLE IV

Simulation Versus Poisson Approx. For $c=31, d=3, l=1, N=127$

\begin{tabular}{|c|c|c|c|c|}
\hline \multirow{2}{*}{$q$} & \multirow{2}{*}{$\begin{array}{c}\text { Offered } \\
\text { Traffic } \\
\text { Rate }\end{array}$} & \multirow{2}{*}{$\begin{array}{l}\text { Total } \\
\text { Variation } \\
\text { Distance }\end{array}$} & \multicolumn{2}{|c|}{ Throughput } \\
\hline & & & Simulation & Poisson \\
\hline \multirow{4}{*}{10} & 10 & 0.708152 & 6.29089 & 3.5892 \\
\hline & 20 & 0.703230 & 7.57373 & 2.4994 \\
\hline & 40 & 0.506468 & 4.34213 & 0.4924 \\
\hline & 80 & 0.405522 & 0.251733 & 0.0032 \\
\hline \multirow{4}{*}{30} & 10 & 0.208599 & 7.79068 & 7.1119 \\
\hline & 20 & 0.279237 & 12.0940 & 10.114 \\
\hline & 40 & 0.305409 & 14.5061 & 10.152 \\
\hline & 80 & 0.236916 & 9.40800 & 4.7837 \\
\hline \multirow{4}{*}{50} & 10 & 0.102966 & 8.4546 & 8.1499 \\
\hline & 20 & 0.151033 & 14.2647 & 13.287 \\
\hline & 40 & 0.191188 & 20.3301 & 17.641 \\
\hline & 80 & 0.177668 & 20.0067 & 15.336 \\
\hline \multirow{4}{*}{80} & 10 & 0.047013 & 8.92707 & 8.7993 \\
\hline & 20 & 0.077785 & 15.9370 & 15.488 \\
\hline & 40 & 0.105190 & 25.3937 & 23.991 \\
\hline & 80 & 0.118728 & 31.9576 & 28.711 \\
\hline
\end{tabular}

approximation) assumes that the number of interferers in each bit is the same.

Unfortunately, the computation for approximation 2 is very complicated. The CPU time increases exponentially as $N$
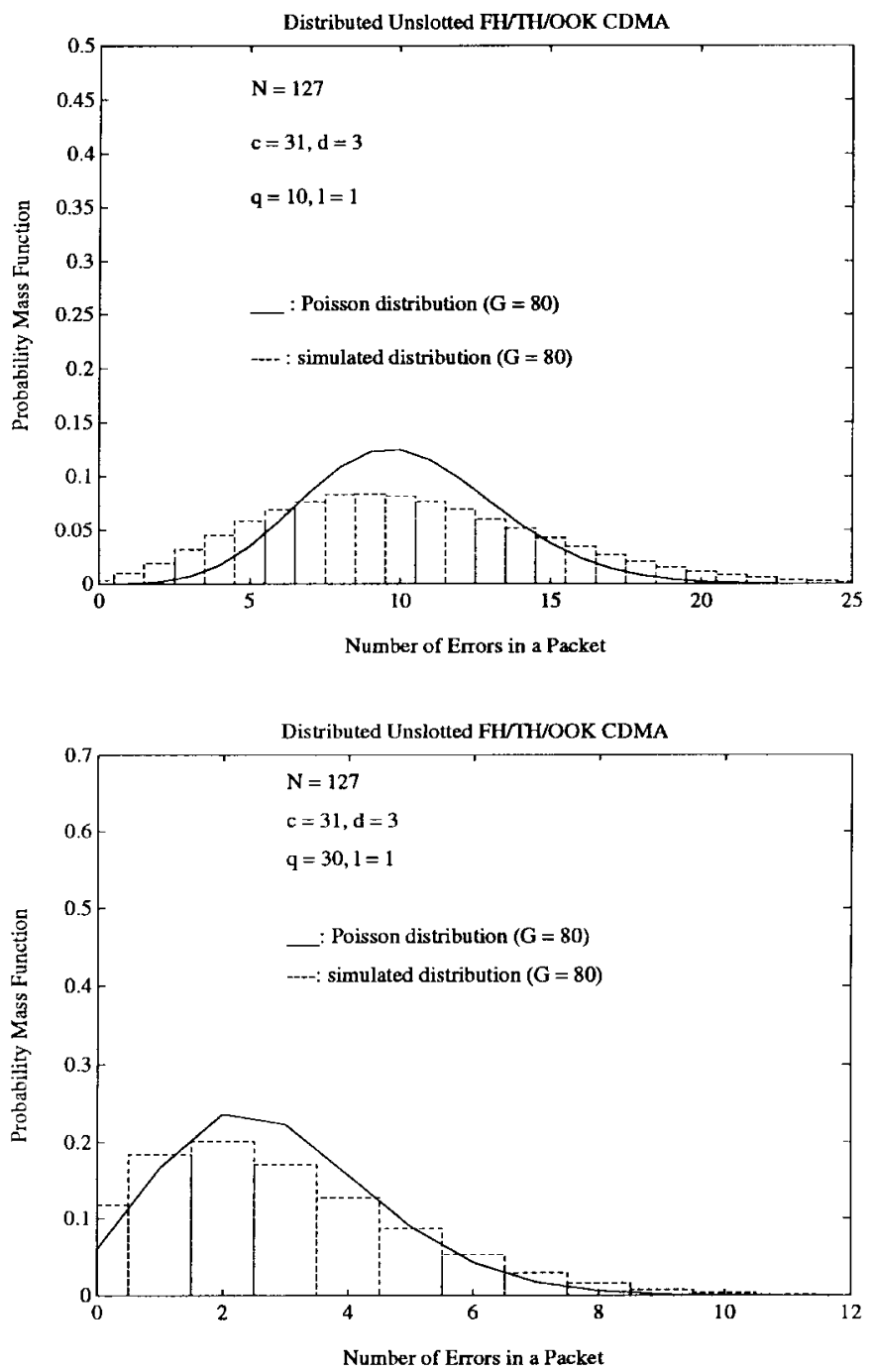

Fig. 8. Probability mass functions for $q=10$ and 30 .

increases. Therefore, approximation 2 is not practical for large $N$. In our case, for $N=127$, the CPU time on a SUN SPARC 400 system is about $100 \mathrm{~h}$ ! However, the computation for approximation 1 (Poisson approximation) is very simple and fast (CPU time of $3 \mathrm{~min}$ for the same parameters; the CPU time for the simulation is about 130 h). Furthermore, it does not depend on $N$. As $q$ increases, the difference between the Poisson approximation and the simulation decreases very quickly, as shown in Fig. 7 and Table IV for the same parameters as above, except that $q$ varies from 10 to 80 . Fig. 8 shows the probability mass functions for $q=10$ and 30, respectively. As $q$ increases, it shows that the Poisson distribution is a good approximation. From the above discussion, approximation 2 can be used in systems with small $N$, and approximation 1 is suitable for systems with large $q$ and large $N$. For example, the standard ATM cell is 53 octets, i.e., 424 bits. Its performance can be evaluated using approximation 1 .

\section{CONCLUSION}

In this paper, we have analyzed the performance of unslotted fiber-optic packet networks. In unslotted systems, the exact solution is very difficult to obtain. Therefore, two approximation 
methods are presented. Furthermore, simulation is performed to verify the accuracy of the results. The numerical results show that approximation 2 can be used in systems with small $N$, and approximation 1 is suitable for systems with large $q$ and large $N$.

\section{REFERENCES}

[1] D. Raychaudhuri, "Performance analysis of random access packetswitched code division multiple access systems," IEEE Trans. Commun., vol. COM-29, pp. 895-901, June 1981.

[2] F. R. K. Chung, J. A. Salehi, and V. K. Wei, "Optical orthogonal codes: Design, analysis and applications," IEEE Trans. Inform. Theory, vol. 35, pp. 595-604, May 1989.

[3] H. Chung and P. V. Kumar, "Optical orthogonal codes-New bounds and an optimal construction," IEEE Trans. Inform. Theory, vol. 36, pp. 866-873, July 1990.

[4] F. Khansefid, H. Taylor, and R. Gagliardi, "Design of $(0,1)$ sequence sets for pulse coded systems," Tech. Rep. CSI-88-03-03, Univ. of Southern California, Mar. 1988.

[5] J. A. Salehi, "Code division multiple-access techniques in optical fiber networks-Part I: Fundamental principles," IEEE Trans. Commun., vol 37, pp. 824-833, Aug. 1989.
[6] J. A. Salehi and C. A. Brackett, "Code division multiple-access techniques in optical fiber networks-Part II: Systems performance analysis," IEEE Trans. Commun., vol. COM-37, pp. 834-842, Aug. 1989.

[7] L. H. Y. Chen, "Poisson approximation for dependent trials," The Annals of Probability, vol. 3, pp. 534-545, Mar. 1975.

[8] R. Arratia, L. Goldstein, and L. Gordon, "Two moments suffice for Poisson approximations: The Chen-Stein methodd," The Annals of Probability, vol. 17, pp. 9-25, Jan. 1989.

[9] M. Yin and V. O. K. Li, "Performance analysis of frequency-hopped spread spectrum packet radio network under the Poisson approximation," in IEEE GLOBECOM, pp. 476-481, 1991.

[10] J. A. B. Tarr, J. E. Wieselthier, and A. Eperemides, "Packet-error probability analysis for unslotted FH-CDMA systems with error-control coding," IEEE Trans. Commun., vol. 38, pp. 1987-1993, Nov. 1990.

Cherng-Shung Hsu (S'88-M'92), for a photograph and biography, see p. 828 of the July 1997 issue of this TransaCTIONS.

Victor O. K. Li (S'80-M'81-SM'86-F'92), for a photograph and biography, see p. 828 of the July 1997 issue of this Transactions. 